\title{
The Influence of Age on the Dynamic Relationship Between End-Tidal Sevoflurane Concentrations and Bispectral Index
}

Luis I. Cortínez, MD*

Iñaki F. Trocóniz, PhD+

Ricardo Fuentes, MD*

Pedro Gambús, MDł

Yung-Wei Hsu, MD§

Fernando Altermatt, MD*

Hernán R. Muñoz, MD, MSc*
BACKGROUND: Age is an important determinant of the pharmacokinetic profile of inhaled anesthetics. The influence of age on the dynamic profile of sevoflurane's effect has not been well described. We performed this study to characterize the influence of age and other covariates on the dynamic relationship between sevoflurane end-tidal concentration $\left(C_{\mathrm{ET}}\right)$ and its effect measured by bispectral index (BIS).

METHODS: Fifty patients, aged 3-71 yr, scheduled for minor surgery were prospectively studied. The BIS and sevoflurane $C_{\mathrm{ET}}$ were continuously measured during the study period. During maintenance of anesthesia and after stable BIS values of 60-65 were obtained, the inspired concentration of sevoflurane was increased to 5 vol \% for 5 min or until BIS $<40$ and then decreased. The dynamic relationship between sevoflurane $C_{\mathrm{ET}}$ and its effect as measured by BIS during this transition period were modeled with an inhibitory $E_{\max }$ model using a population pharmacokinetic-pharmacodynamic approach with NONMEM V. A predictive check method was used to validate the final model.

RESULTS: The sensitivity to sevoflurane's effect as measured by BIS expressed in the $C_{50}$ [steady-state $C_{\mathrm{ET}}$ eliciting half of maximum response $\left(I_{\text {max }}\right)$ ] increased with age. The speed of change of sevoflurane's effect, expressed as the effect-site equilibration half-life $\left(t_{1 / 2} k_{\mathrm{e} 0}\right)$, increased at older ages. The predictive check analysis confirmed the adequacy of the model.

CONCLUSIONS: Age significantly affects the dynamic relationship between sevoflurane $C_{\mathrm{ET}}$ and its effect measured with BIS.

(Anesth Analg 2008;107:1566-72) olatile anesthetics exert their hypnotic effect in the brain. Normally, end-tidal concentrations $\left(C_{\mathrm{ET}}\right)$ of these drugs are used as indirect measures of their brain concentration at stable anesthetic depths. However, during transitions of the anesthetic depth level, delays between $C_{\mathrm{ET}}$ and the concentration in the effect-site $\left(C_{\mathrm{e}}\right)$ occur. ${ }^{1}$ Pharmacokinetic-pharmacodynamic (PK-PD) modeling has allowed the quantification of these delays incorporating the plasma-effect site equilibration rate constant $\left.\left(k_{\mathrm{e}}\right)\right)^{2}$ This has led to a greater understanding of the clinical properties of these anesthetics in terms of onset, dosing, and offset of effect.

From the *Departamento de Anestesiología, Escuela de Medicina, Pontificia Universidad Católica de Chile. Santiago, Chile; tDepartment of Pharmacy; School of Pharmacy; University of Navarra; Pamplona, Spain; ‡Department of Anesthesiology, Hospital CLINIC, Universidad de Barcelona, CIBERehd, IDIBAPS, Barcelona, Spain; and §Department of Anesthesiology, Mackay Memorial Hospital, Taiwan.

Accepted for publication May 16, 2008.

Address correspondence and reprint requests to Dr. Luis I. Cortínez, Departmento de Anestesiología, Hospital Clínico U.C., Marcoleta 367, Santiago, Chile. Address e-mail to licorti@med. puc.cl.

Copyright (C 2008 International Anesthesia Research Society DOl: 10.1213/ane.0b013e318181f013
The influence of age on the dynamic relationship between the $C_{\mathrm{ET}}$ and the hypnotic effect of volatile anesthetics has not been explored with PK-PD modeling. In theory, a decrease in the proportion of cardiac output directed to the vessel-rich tissues ${ }^{3}$ and an increase in tissue and blood solubility of inhaled anesthetics with age ${ }^{4}$ should result in progressively slower onset and offset of the effect measured by bispectral index (BIS). These assumptions, however, do not consider possible influences of cortical and subcortical neuronal dynamics that might be affected by age. ${ }^{5}$

The aim of this study was to characterize the influence of age and other covariates on the dynamic relationship between sevoflurane $C_{\mathrm{ET}}$ and its effect measured with BIS [Aspect A-2000 BIS ${ }^{\circledR}$ monitor (version $\mathrm{XP})]$.

\section{METHODS}

After IRB approval (School of Medicine, Pontificia Universidad Católica, Santiago, Chile) and obtaining written informed consent, 50 patients with ASA physical status I or II, aged 3-71 yr, were prospectively studied. All patients were unpremedicated and scheduled to undergo general anesthesia for minor elective 
surgery. Exclusion criteria were any known cerebrovascular disease, long- or short-term (within the previous $24 \mathrm{~h}$ ) intake of any drug acting in the central nervous system, and any known adverse effect to the study drugs.

In the operating room, after standard monitoring, anesthesia was induced either by sevoflurane inhalation or by an IV bolus of propofol $1-2 \mathrm{mg} / \mathrm{kg}$. If tracheal intubation was part of the anesthetic plan, fentanyl 1-3 $\mu \mathrm{g} / \mathrm{kg}$ and rocuronium $0.6 \mathrm{mg} / \mathrm{kg}$ were also administered. After induction, the airway was secured either with a laryngeal mask airway or with an endotracheal tube, and patients' lungs were mechanically ventilated to obtain an end-tidal carbon dioxide of approximately $35 \mathrm{~mm} \mathrm{Hg}$. Peak airway pressure was maintained under $20 \mathrm{~cm} \mathrm{H}_{2} \mathrm{O}$ and confirmation that no gas leaked by auscultation was also checked at this stage. Maintenance of anesthesia was with sevoflurane and oxygen. No more hypnotic drugs, opioids, or muscle relaxants were given until the study was finished.

During the maintenance period of anesthesia, patients were monitored with the Aspect A-2000 BIS ${ }^{\circledR}$ monitor (version XP). The skin was prepared to ensure low impedance and good quality of signal and the sensors were attached according to the manufacturer's recommendations. The pediatric sensors and the QUATRO sensors were used for children $(\leq 15 \mathrm{yr})$ and adult patients, respectively, and the smoothing time period was set at $15 \mathrm{~s}$.

The study was performed during maintenance of anesthesia either before or after surgery. Patients who received propofol and/or fentanyl during induction were studied at the end of surgery after a period of at least 45-min from induction to minimize the influence of propofol or fentanyl on BIS. During the study period, the patients were kept undisturbed with the operating room in silence and without any surgical or tactile stimulation. No arterial blood pressure measurements were allowed at this time. Fresh oxygen flow was set at $5 \mathrm{~L} / \mathrm{min}$ and sevoflurane concentrations were gradually decreased to obtain relatively stable BIS values of $60-65$. After 5 min of stable BIS values (baseline period), sevoflurane inspired concentrations were then increased to $5 \mathrm{vol} \%$ for a maximum of $5 \mathrm{~min}$ or until BIS reached values $<40$. The vaporizer was then closed until BIS returned to basal values (60-65). At this point, the study was finished and anesthesia continued according to the anesthesiologist's criteria. During the study period, BIS values and expiratory sevoflurane concentrations, measured by a Datex Capnomac monitor (Datex, Helsinski, Finland), were manually recorded every $10 \mathrm{~s}$.

A parametric approach was used to model the effect of sevoflurane measured with BIS. In this approach, it was assumed that $C_{\mathrm{ET}}$ sevoflurane reflects its plasma concentration $\left(C_{p}\right)$ and that predicted sevoflurane concentrations in the effect compartment were linked to $C_{p}$ through a first-order process as it is shown in Eq. 1.

$$
\frac{d C_{\mathrm{e}}}{d t}=k_{\mathrm{e} 0} \times\left(C_{\mathrm{p}}-C_{\mathrm{e}}\right)
$$

where $d C_{\mathrm{e}} / d t$ represents the rate of change of $C_{\mathrm{e}}$ and $k_{\mathrm{e} 0}$ is the first-order rate constant of equilibrium between plasma and biophase. The relation between the $C_{\mathrm{e}}$ and the BIS effect was mathematically modeled. The PD model used to fit the BIS effect data was the inhibitory sigmoidal $I_{\max }$ model (Eq. 2).

$$
E=E_{0} \times\left[1-I_{\max } \times \frac{C_{\mathrm{e}}^{\gamma}}{C_{\mathrm{e}}^{\gamma}+C_{50}^{\gamma}}\right]
$$

where $E$ is the BIS value being measured, $E_{0}$ is the BIS value in the absence of sevoflurane, $I_{\max }$ is the maximum decrease in the response that the sevoflurane can elicit and can range from 0 to $1, C_{50}$ is the steady-state plasma concentration of sevoflurane eliciting half of $I_{\max }$ and $\gamma$ is the steepness of the concentrationresponse curve. The model parameters were estimated using a population analysis with NONMEM version V (Globomax LLC, Hanover, MD). ${ }^{6}$ Interindividual variability was modeled using an exponential model (Eq. 3).

$$
P_{i}=P_{\mathrm{TV}} \times e^{\eta i}
$$

where $P_{i}$ is the parameter value $\left(E_{0}, I_{\max }, \gamma\right.$, or $\left.C_{50}\right)$ in the $i$ th patient, $P_{\mathrm{TV}}$ is the typical value of the parameter in the population, and $\eta$ is a random variable with a mean of 0 and variance of $\omega^{2}$ Residual intraindividual variability was modeled with an additive error model. The FOCE with INTERACTION estimation method, together with the subroutine ADVAN6 $\mathrm{TOL}=5$, was used during the analysis.

Model selection was based mainly on the inspection of goodness-of-fit plots and the precision of the parameter estimates. The minimum value of the objective function $[-2 \cdot \log$-likelihood $(-2 \mathrm{LL})]$ provided by NONMEM served as a guide during model building. For two nested models, a decrease in -2LL of 3.84 or 6.63 points for an added parameter is considered significant at the 0.05 or 0.01 level, respectively.

Results from the population models are presented as parameter estimates, together with the corresponding relative standard error. These last values are computed as the ratio between the standard error and the estimate of the parameter. Interindividual variability is expressed as coefficient of variation [CV (\%)].

In our modeling approach, we first developed the base population model, which better described the data. At this stage of the analysis, no covariates were included in the model. The effects of age, weight, gender, lean body mass height, and heart rate were then explored. Allometric scaling of weight was tested at this stage. Covariates selection was performed 
Table 1. Demographic and General Data

Age (yr)

Female/male ratio $(n)$

Weight $(\mathrm{kg})$

Height $(\mathrm{cm})$

Baseline mean arterial pressure (mm Hg)

Baseline cardiac rate (bpm)

Baseline BIS value

Minimum BIS value

Duration of study period (min)

$n=50$; values are mean \pm SD (range).

$\mathrm{BIS}=$ bispectral index

using the GAM method ${ }^{7}$ implemented in Xpose 3.1 program (a population PK-PD model building program for NONMEM found at http://sourceforge.net). ${ }^{8}$ To determine the relevant covariates of the final model, a forward inclusion and backward elimination approach was used in consecutive NONMEM runs. ${ }^{9}$ During the forward inclusion and backward elimination approaches, the levels of significance used were 0.05 and 0.01 , respectively.

The final population model was evaluated by visual predictive check ${ }^{10}: 100$ datasets of the same characteristics of the original dataset were simulated using the final population PD model. The 5th, 50th, and 95th percentiles of the BIS versus time simulated profiles were first computed for patients (i) younger than 20 years, (ii) between 20 and $50 \mathrm{yr}$, and (iii) older than 50 $\mathrm{yr}$, and then plotted together with the corresponding raw data.

The impact of the selected covariates on the sevoflurane dynamic profile was further explored using simulations based on the selected model as follows: The average sevoflurane $C_{\mathrm{ET}}$ time profile was calculated from all the individual time profiles measured in our patients. This average sevoflurane time profile was then used to generate typical population BIS time profiles for different representative values of the selected covariates.

\section{RESULTS}

All patients $(n=50)$ were included in the analysis. Demographic and general data observed during the study period are summarized in Table 1 . The observed time course of sevoflurane $C_{\mathrm{ET}}$ and BIS is shown in Figure 1.

The selected base population model consisted of a sigmoidal model with interindividual variability estimated for $C_{50}, \gamma$, and $k_{\mathrm{e} 0}$. Since we did not measure BIS before sevoflurane administration, we fixed E0 to 97, an allowed a $5 \%$ interindividual variability for this parameter, and $I_{\max }$ was fixed to 1 . In this model, the time course of $C_{p}$ was described by linear interpolation. The parameter estimates of the base population model are shown in Table 2, where it can be observed that all of the parameters were estimated with very good precision.
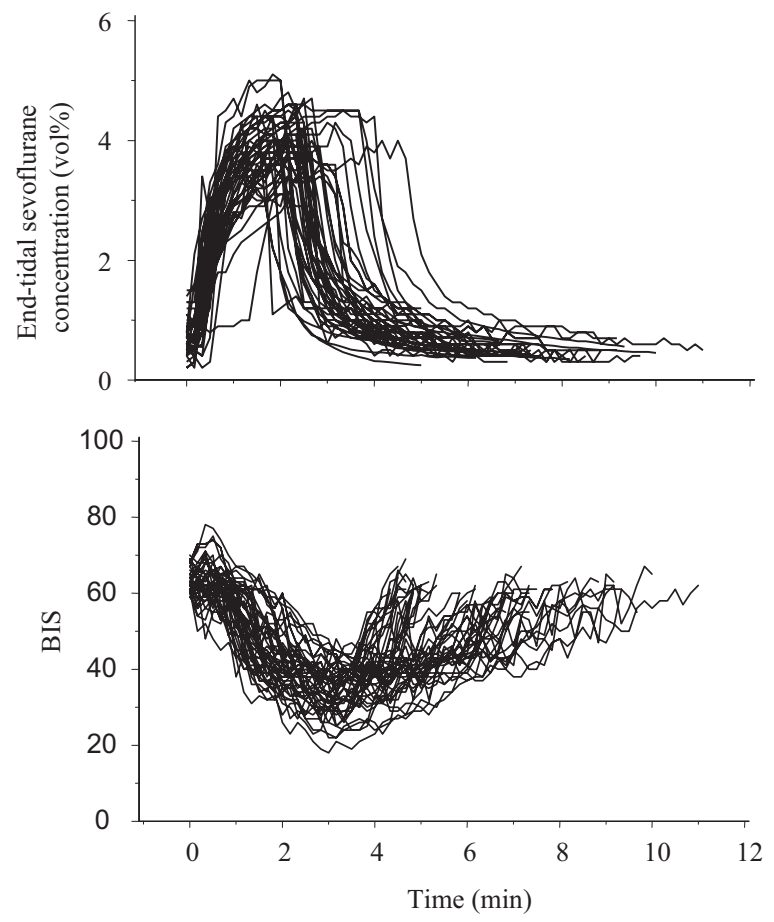

Figure 1. Time course of the measured end-tidal sevoflurane concentrations values (top panel) and time course of the measured bispectral index values (bottom panel) in all patients.

Table 2. Population Pharmacokinetics/Pharmacodynamic Parameters of Sevoflurane in the Final Base Population Model

\begin{tabular}{lcc}
\hline Parameter & $\begin{array}{c}\text { Typical population } \\
\text { value }\left(P_{\mathrm{TV}}\right)\end{array}$ & $\begin{array}{c}\text { Interindividual } \\
\text { variability }\left(\omega^{2}\right)\end{array}$ \\
\hline$E_{0}(\mathrm{BIS})$ & 97 fixed & 5 fixed \\
$C_{50}(\%)$ & $1.5(0.04)$ & $27.7(0.17)$ \\
$I_{\mathrm{max}}$ & 1 fixed & - \\
$k_{\mathrm{e} 0}\left(\mathrm{~min}^{-1}\right)$ & $0.38(0.05)$ & $36.6(0.22)$ \\
$\gamma$ & $1.2(0.06)$ & $36.7(0.23)$ \\
Residual error & $4.6(0.06)$ & - \\
$\quad(\mathrm{SD})$ & & - \\
\hline
\end{tabular}

Minimum value of the objective function $=8728$.

Estimates are presented together with the corresponding relative standard errors in parenthesis. Estimates of interindividual variability are expressed as CV (\%). Parameters are defined in the text.

$\mathrm{SD}=$ standard deviation; BIS = bispectral index.

During the covariate model selection, only age was found to be a significant covariate for $C_{50}$ and $k_{\mathrm{e} 0}(P<$ $0.01)$. This variable was explored as a continuous and as a categorical variable (children $<10 \mathrm{yr}=1$; patients $>10 \mathrm{yr}=2$ ) during this process. The relationship between these parameters and their covariates is shown in Figure 2. The typical parameter values of $C_{50}$ and $k_{\mathrm{e} 0}$ for the median age subject are $1.49 \%$ and 0.383 $\min ^{-1}$, values very close to the ones obtained in the base population model and listed in Table 2.

Table 3 lists the model parameter estimates of the final selected model where again parameter precision was high. Figure 3 shows two goodness-of-fit plots consisting of the relationship between the observations and the individual model predictions (left 
Figure 2. Two scatter plots of the parameter-covariate relationships included in the final model. Left panel: $C_{50}$-age relationship. Right panel: $k_{\mathrm{e} 0}$-age relationship. The solid lines represent the covariate model predictions based on the typical values of the parameters. The good quality of the fit corroborates the parameterscovariate relationship used in the model.
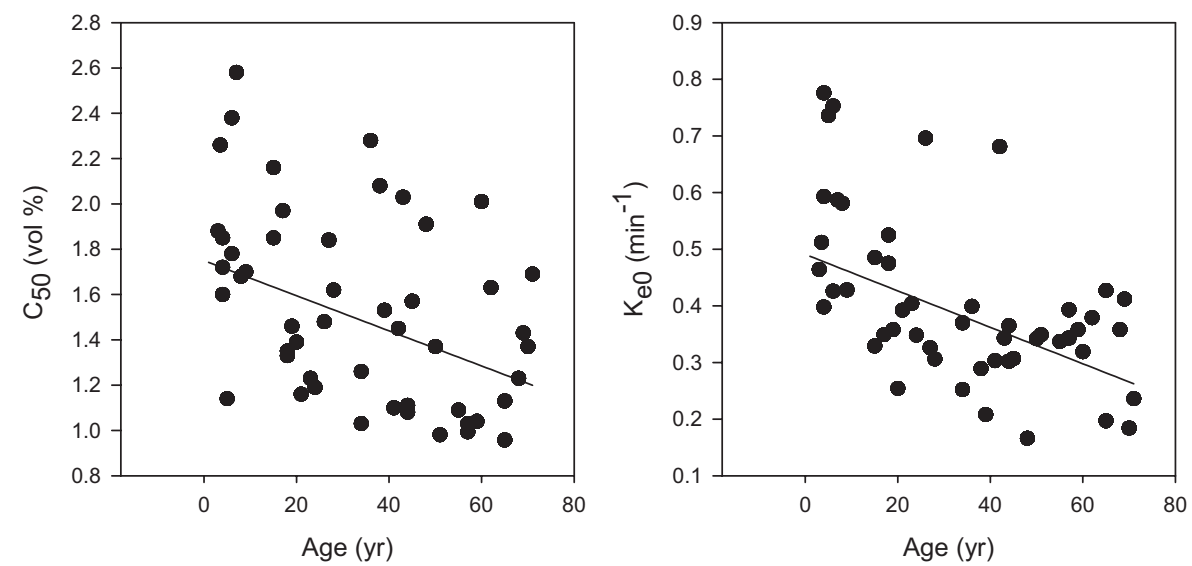

Table 3. Population Pharmacokinetics/Pharmacodynamic Parameters of Sevoflurane in the Final Covariate Population Model

\begin{tabular}{lcr}
\hline \multicolumn{1}{c}{ Parameter } & Typical population value $\left(P_{\mathrm{TV}}\right)$ & Interindividual variability $\left(\omega^{2}\right)$ \\
\hline$E_{0}(\mathrm{BIS})$ & 97 fixed & 5 fixed \\
$C_{50}=\theta_{1} \times\left[1+\theta_{2} \times(\right.$ age-33.5) & $\theta_{1}(\%)=1.49(0.04)$ & $25.3(0.17)$ \\
$I_{\max }$ & $\theta_{2}\left(\mathrm{age}^{-1}\right)=-0.0052(0.31)$ & - \\
$k_{\mathrm{e} 0}=\theta_{3} \times\left[1+\theta_{4} \times(\right.$ age-33.5) & 1 fixed & $31(0.22)$ \\
$\gamma$ & $\theta_{3}\left(\mathrm{~min}^{-1}\right)=0.383(0.05)$ & $36.7(0.24)$ \\
Residual error (SD) & $\theta_{4}\left(\mathrm{age}^{-1}\right)=-0.0084(0.23)$ & - \\
\hline
\end{tabular}

Minimum value of the objective function $=8706$.

Estimates are presented together with the corresponding relative standard error in parenthesis. Estimates of interindividual variability are expressed as CV (\%). Parameters are defined in the text. $\mathrm{SD}=$ standard deviation; BIS = bispectral index.

Figure 3. Goodness of fit plots corresponding to the final model. Left panel: Relationship between individual model predictions and observations. The solid line represents the line of identity. Right panel: Ratio between observed and individual model predicted bispectral index values versus time. Horizontal line represents the perfect prediction $(y=1)$.
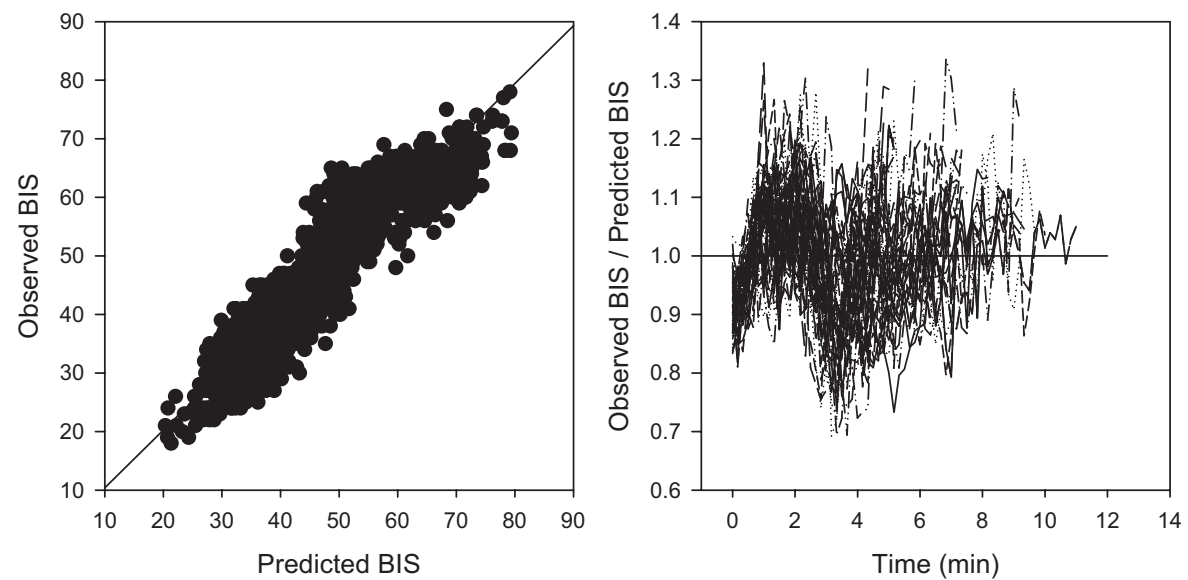

panel), and the ratio between individual model predictions and observed values versus time (right panel). No major tendencies are present in the graphics indicating a very good model performance. Figure 4 shows the relationship between the sevoflurane $C_{\mathrm{ET}}$ and BIS (left panel), and the relationship between the predicted sevoflurane $C_{\mathrm{e}}$ and BIS (right panel) where an adequate collapse of the hysteresis loop is observed. Table 4 shows typical estimates of $t_{1 / 2} k_{\mathrm{e} 0}$ values according to age, where $t_{1 / 2} k_{\mathrm{e} 0}=\ln 2 / k_{\mathrm{e} 0}$.

Results from the visual predictive check are shown in Figure 5, which shows that the final population model adequately captures the mean tendencies of the data and their dispersion.

Finally, the effect of age in BIS time profiles evaluated by computed simulations is presented in Figure
6 . The values of age used for the simulations correspond to the 5th, 50th, and 95th percentiles of the distribution with the studied population.

\section{DISCUSSION}

The main finding of this study is that the dynamic relationship between sevoflurane $C_{\mathrm{ET}}$ and its effect measured with BIS are affected by age. Although the sensitivity to sevoflurane measured with BIS effect expressed in the $C_{50}$ increases with age, the speed of change of sevoflurane's effect expressed in the $t_{1 / 2} k_{\mathrm{e} 0}$ is slower at older ages.

Katoh et al. ${ }^{11}$ analyzed the influence of age on the hypnotic requirement of sevoflurane using BIS and spectral edge frequency. In this study, 96 patients 

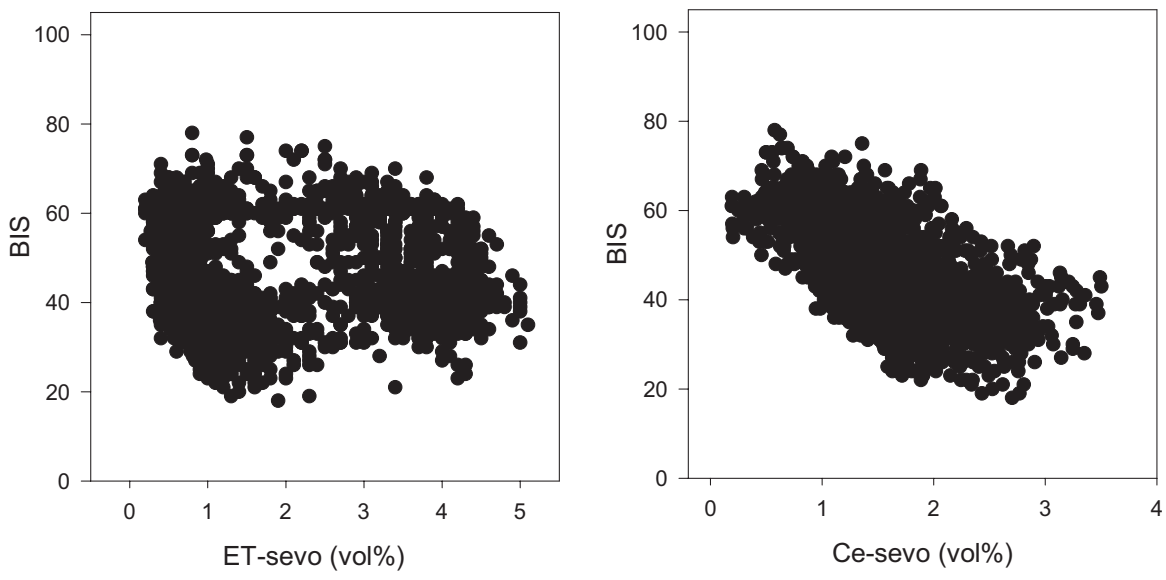

Figure 4. Left panel: Relationship between end-tidal sevoflurane concentration and bispectral index (BIS) revealing a hysteresis loop. Right panel: Relationship between the effect-site sevoflurane concentrations against BIS. The collapse of the hysteresis loop is observed.
Table 4. Typical $t_{1 / 2} k_{\mathrm{e} 0}$ Values According to Age

\begin{tabular}{cc}
\hline Age $(\mathrm{yr})$ & $t_{1 / 2} k_{\mathrm{e} 0}(\min )$ \\
\hline 5 & 1.46 \\
25 & 1.69 \\
50 & 2.1 \\
75 & 2.78 \\
\hline
\end{tabular}

Estimates are derived from the typical values of the final covariate population model parameters.

from 18 to $80 \mathrm{yr}$ were sedated with different concentrations of sevoflurane and the depth of sedation was assessed with the Observer Assessment of Alertness and Sedation rating scale. The authors showed that age reduced MAC-awake, but did not change BIS and $95 \%$ spectral edge frequency associated with response to a verbal command. In agreement with this and other previous studies, ${ }^{11-13}$ our analysis using an effect compartment model linked with an $I_{\max }$ model showed that age significantly reduced the requirements of sevoflurane. The $C_{50}$ estimated is comparable to previous values reported in adult patients. ${ }^{1,14}$
The slower speed of sevoflurane's effect observed in elderly patients compared with younger patients (Table 4) is consistent with the decrease in the proportion of cardiac output directed to the vessel-rich tissues $^{3}$ and the increase in tissue and blood solubility of inhaled anesthetics with age. ${ }^{4}$ As it can be seen in the simulation analysis (Fig. 6), age differences in the speed of sevoflurane's effect during induction are less evident than during recovery. This is probably explained by the greater sensitivity observed at older ages, since the same sevoflurane dose scheme was used in all patients for this simulation. Our results showing longer times for sevoflurane end-tidal brain equilibration in elderly patients is also consistent with the results reported for other drugs, such as remifentanil, where blood-brain equilibration times increase nearly threefold in elderly patients compared with younger patients. ${ }^{15}$ The current results, however, cannot be extrapolated to other more soluble volatile anesthetics, since the alveolar concentration of a

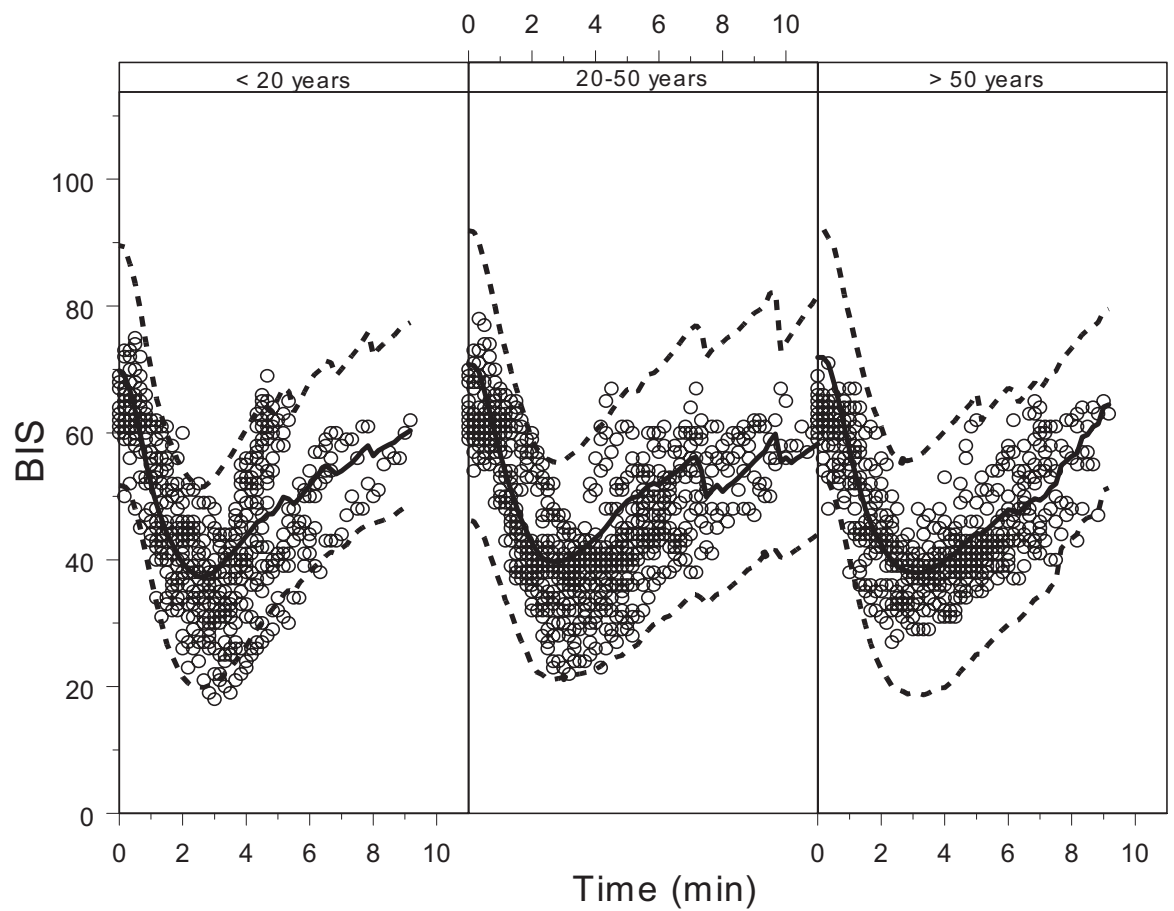

Figure 5. Results from the visual predictive check. Lines represents the 5th, 95th (dashed), and 50th (solid) percentiles bispectral index versus time profiles computed from one hundred model based simulated datasets. Open circles represent raw data. 
Figure 6. Model predicted bispectral index versus time profiles for typical 4 yr (black solid line), 34 yr (solid gray line), and $68 \mathrm{yr}$ (dashed line) old patients.

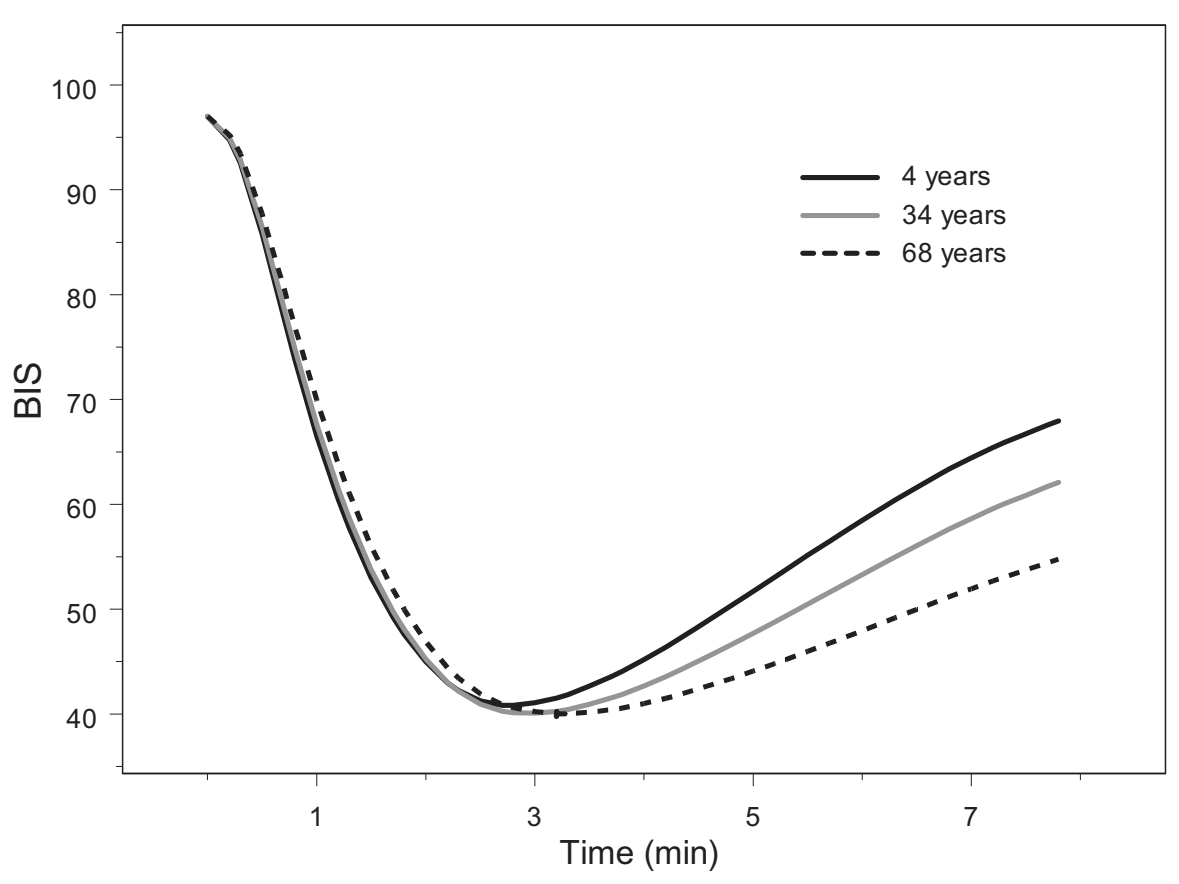

highly soluble agent is much more influenced by changes in cardiac output. This means that the decrease in cardiac output associated with aging might result in the faster increase of the alveolar concentration of a soluble anesthetic and, consequently, faster speed of anesthetic effect might be observed in elderly patients with these agents. ${ }^{3}$

It should be considered that we assumed that the sevoflurane $C_{\mathrm{ET}}$ reflected its plasma concentration. This is important because the $t_{1 / 2} k_{\mathrm{e} 0}$ estimates not only represent the blood-brain equilibration time but also the end-tidal to plasma equilibration time. In addition, since BIS was used as the measured response, time delays in BIS calculation will also be represented in the $t_{1 / 2} k_{\mathrm{e} 0}$ estimates.

Making the $C_{p}$ of volatile anesthetics available improves the titration of these drugs during clinical anesthesia. ${ }^{16}$ Using children and adult data, we were able to demonstrate that the observed $k_{\mathrm{e} 0}$ variability in this population produced significant changes in sevoflurane's effect. For example, the $t_{1 / 2} k_{\mathrm{e} 0}$ in a typical patient of $75 \mathrm{yr}$ was found to be almost twice that observed in a typical patient of $5 \mathrm{yr}$ (Table 4). The clinical implication of this difference is clearly shown in the slower changes of offset of sevoflurane's effect in elderly patients (Fig. 6). Since this is the first study analyzing the influence of age and other covariates in sevoflurane $t_{1 / 2} k_{\mathrm{e} 0}$, the inclusion of our findings would be helpful in refining sevoflurane predictive models.

One limitation of this study is that age-related changes in the electroencephalogram (EEG) might influence our results, since BIS has been shown to be associated with age. ${ }^{17}$ However, most maturational EEG changes occur early in life ( $<1 \mathrm{yr})$. In Davidson et al.'s study, ${ }^{18}$ the authors describe that awake BIS values in children between 1 and 15 yr were similar to values reported in adult patients and that the relationship between sevoflurane concentration and BIS response during emergence of anesthesia in children between 1 and 15 yr was also consistent with adult studies. We arbitrarily excluded children $<3$ yr to avoid potential influences of maturational EEG changes. In addition, since we only studied 11 patients $<10 \mathrm{yr}$, it might also be argued that more children would have been required to adequately represent the important physiological changes that occur in children.

We conclude that age significantly affects the dynamic relationship between sevoflurane $C_{\mathrm{ET}}$ and its effect measured with BIS. Future development of an on-line available sevoflurane PK-PD model, including this variable, might lead to better predictability and control of sevoflurane's effect in our patients.

\section{REFERENCES}

1. Rehberg B, Bouillon T, Zinserling J, Hoeft A. Comparative pharmacodynamic modeling of the electroencephalographyslowing effect of isoflurane, sevoflurane, and desflurane. Anesthesiology 1999;91:397-405

2. Sheiner LB, Stanski DR, Vozeh S, Miller RD, Ham J. Simultaneous modeling of pharmacokinetics and pharmacodynamics: application to d-tubocurarine. Clin Pharmacol Ther 1979;25: 358-71

3. Eger EI. Uptake and Distribution. In: Miller RD, ed. Miller's anesthesia. 6th ed. Philadelphia: Elsevier, 2005:131-54

4. Lerman J, Schmitt-Bantel BI, Gregory GA, Willis MM, Eger EI II. Effect of age on the solubility of volatile anesthetics in human tissues. Anesthesiology 1986;65:307-11

5. Torres F, Anderson C. The normal EEG of the human newborn. J Clin Neurophysiol 1985;2:89-103

6. Beal SL. NONMEM Users Guide, parts I and II. San Francisco: University of California, 1980

7. Mandema JW, Verotta D, Sheiner LB. Building population pharmacokinetic-pharmacodynamic models. I. Models for covariate effects. J Pharmacokinet Biopharm 1992;20:511-28 
8. Jonsson EN, Karlsson MO. Xpose-an S-PLUS based population pharmacokinetic/pharmacodynamic model building aid for NONMEM. Comput Methods Programs Biomed 1999;58:51-64

9. Jonsson EN, Karlsson MO. Automated covariate model building within NONMEM. Pharm Res 1998;15:1463-8

10. Holford N. The visual predictive check-superiority to standard diagnostic (Rorschach) plots. Presented at the 14th meeting of the Population Approach Group in Eure. Available at http:/ / www.page-meeting.org/default.asp?abstract=738. 2005

11. Katoh T, Bito H, Sato S. Influence of age on hypnotic requirement, bispectral index, and 95\% spectral edge frequency associated with sedation induced by sevoflurane. Anesthesiology 2000;92:55-61

12. Katoh T, Suguro Y, Ikeda T, Kazama T, Ikeda K. Influence of age on awakening concentrations of sevoflurane and isoflurane. Anesth Analg 1993;76:348-52

13. Mapleson WW. Effect of age on MAC in humans: a metaanalysis. Br J Anaesth 1996;76:179-85

14. Olofsen E, Dahan A. The dynamic relationship between endtidal sevoflurane and isoflurane concentrations and bispectral index and spectral edge frequency of the electroencephalogram. Anesthesiology 1999;90:1345-53
15. Minto CF, Schnider TW, Shafer SL. Pharmacokinetics and pharmacodynamics of remifentanil. II. Model application. Anesthesiology 1997;86:24-33

16. Kennedy RR, French RA, Gilles S. The effect of a model-based predictive display on the control of end-tidal sevoflurane concentrations during low-flow anesthesia. Anesth Analg 2004;99: 1159-63

17. Wodey E, Tirel O, Bansard JY, Terrier A, Chanavaz C, Harris R, Ecoffey C, Senhadji L. Impact of age on both BIS values and EEG bispectrum during anaesthesia with sevoflurane in children. $\mathrm{Br}$ J Anaesth 2005;94:810-20

18. Davidson AJ, McCann ME, Devavaram P, Auble SA, Sullivan LJ, Gillis JM, Laussen PC. The differences in the bispectral index between infants and children during emergence from anesthesia after circumcision surgery. Anesth Analg 2001;93:326-30 